\title{
On the receiving end: have patient perceptions of the side-effects of cancer chemotherapy changed since the twentieth century?
}

\author{
Janette L. Vardy ${ }^{1,2,3}$ (1) $\cdot$ Andre Liew $^{1} \cdot$ Anne Warby $^{3} \cdot$ Alexander Elder $^{1} \cdot$ Itay Keshet $^{3} \cdot$ Rhonda Devine $^{3}$. \\ Calina Ouliaris $^{1} \cdot$ Corrinne Renton $^{3} \cdot$ Martin H. N. Tattersall $^{1,3} \cdot$ Haryana M. Dhillon $^{3,4}$
}

Received: 12 August 2021 / Accepted: 31 December 2021 / Published online: 11 January 2022

(C) The Author(s) 2022

\begin{abstract}
Background Studies in 1983 and 1993 identified and ranked symptoms experienced by cancer patients receiving chemotherapy. We repeated the studies to obtain updated information on patient perceptions of chemotherapy-associated symptoms. Patients and methods A cross-sectional interview and patient-reported outcome questionnaires were administered to outpatients receiving chemotherapy. Patients selected from 124 cards to identify and rank the severity of physical and nonphysical symptoms they had experienced and attributed to chemotherapy (primary endpoint). The patient's medical oncologist and primary chemotherapy nurse were invited to rank the five symptoms they believed the patient would rank as their most severe. We analysed the association of symptoms and their severity with patient demographics, chemotherapy regimen, and patient-reported outcomes. Results were compared to the earlier studies.

Results Overall, 302 patients completed the interview: median age 58 years (range 17-85); 56\% female; main tumour types colorectal 81 (27\%), breast 67 (22\%), lung 49 (16\%); 45\% treated with curative intent. Most common symptoms (reported by $>50 \%$ ) were: alopecia, general weakness, effects on family/partner, loss of taste, nausea, fatigue, difficulty sleeping, effects on work/home duties, and having to put life on hold. The most severe symptoms (ranked by $>15 \%$ in top five) were: concern about effects on family/partner, nausea, fear of the future, fatigue, not knowing what will happen, putting my life on hold, and general weakness. Perceptions of doctors and nurses of patients' symptom severity closely matched patients' rankings. Conclusions Compared to earlier studies, there was an increase in non-physical concerns such as effects on family and future, and a decrease in physical symptoms, particularly vomiting, but nausea, fatigue and general weakness remained bothersome. Highlights • Symptoms related to chemotherapy have changed over time, likely due to less toxic regimens and improvements in supportive care.

- Effects on family/partner, fear of the future, not knowing what will happen, and "life on hold" were major issues for patients.

- Vomiting has decreased but nausea, fatigue and general weakness remain common symptoms for chemotherapy patients.
\end{abstract}

Keywords Symptoms $\cdot$ Chemotherapy $\cdot$ Chemotherapy side-effects $\cdot$ Patient-reported outcomes

Rhonda Devine and Martin H. N. Tattersall is deceased. This paper are dedicated to his/her memory.

Janette L. Vardy

janette.vardy@sydney.edu.au

1 Faculty of Medicine and Health, University of Sydney, Sydney, Australia

2 Concord Cancer Centre, Concord Repatriation General Hospital, Hospital Rd., Concord, NSW 2137, Australia

3 Centre for Medical Psychology and Evidence-based Decision-making, University of Sydney, Sydney, Australia

4 School of Psychology, Faculty of Science, The University of Sydney, Sydney, Australia

\section{Background}

Two studies published in 1983 [1] and 1993 [2] identified and ranked symptoms experienced by patients with cancer who were receiving chemotherapy. In 1983, 99 patients who were receiving chemotherapy reported that non-physical side effects constituted $54 \%$ of the 15 most severe symptoms; these included the thought of coming for treatment, length of time treatment takes, and having to have a needle. Major physical side effects were vomiting, nausea and hair loss. When physical and non-physical categories were combined, vomiting, nausea and hair loss remained the most severe [1]. 
A decade later, patients reported a reduction in the severity of some symptoms, particularly vomiting, and a shift from concerns about physical to psychosocial issues. Nausea was the most severe symptom followed by tiredness and alopecia. Vomiting was of lesser concern, reflecting the introduction of 5HT3 antagonist anti-emetics. Concern about the effect on friends and family increased in rankings from $10^{\text {th }}$ to $3^{\text {rd }}$. In both studies, differences were seen in the symptoms experienced and their severity, based on chemotherapy regimen, age, and sex [2].

Similar methodology was applied to 100 French patients with advanced cancer, recruited between 1998 and 2000 [3]. The side-effect identified as most common and severe was "affects my family or partner", followed by "loss of hair", then "constantly tired".

Since 2000, there have been major changes in chemotherapy and in supportive care to manage side-effects and symptoms, increased patient involvement in cancer care decisions, and people wanting more information about their treatment including side-effects [4-6]. Here, we provide information on patient perceptions of chemotherapy-associated symptoms in people being treated with modern chemotherapy and supportive care including anti-emetic regimens.

\section{Methods}

This cross-sectional survey was conducted as a face-to-face interview with patient-reported outcome measures (PROM) on one occasion when patients were attending out-patient medical oncology clinics at two metropolitan teaching hospitals (one of which was included in the prior studies) and one regional hospital, between May 2008 and October 2016. Patients with any stage cancer, who were receiving chemotherapy, were invited to participate in the study. Eligibility criteria included a diagnosis of invasive cancer, currently receiving chemotherapy for a solid malignancy and completion of at least one cycle, and sufficient English to complete PROM.

\section{Procedures}

The methodology used in the previous studies [1,2], with additional questions, was retained to maximise validity of comparisons. Additional symptoms were added to the original list by an expert group that consisted of two medical oncologists, a senior cancer nurse, social worker, clinical psychologist, and behavioural scientist. The additional items (highlighted in Table 1) were piloted on 10 patients to assess understanding and construct validity.

The patient interview was scripted and was conducted by trained research assistants. The procedure was as described in the previous two studies $[1,2]$. In brief, two sets of white cards were prepared. Group A listed physical side-effects of chemotherapy $(n=69)$ and Group B listed non-physical side-effects of chemotherapy $(n=55)$ (Table 1). Both card sets were shuffled, and Group A cards were presented first, one at a time. The participant was asked to select all cards that described a symptom they had experienced and that they attributed to their current chemotherapy. They were then asked to rank the selected cards in order of severity. This process was repeated for Group B cards. The five highest ranked cards from each group were combined and the patient was asked to select and rank the five most severe symptoms regardless of group. Five points were allocated to symptoms ranked as the most severe, decreasing to one point for symptoms ranked as least severe. The allocated points were used to generate an overall ranking of symptom severity. The patient's medical oncologist and a nurse closely involved in their care were also asked to rank the five symptoms they believed that each patient would rank as their most severe. They were given a separate list of physical and non-physical symptoms, grouped by systems or domains, and asked firstly to rank the top 5 for each list. They were then asked to rank the top 5 across both symptom lists.

Demographic, disease, and treatment characteristics were collected from the participant and their medical record. The research assistant rated the participant's performance status based on European Co-operative Oncology Group (ECOG) criteria [7], and determined their Colinet Simplified Comorbidity Score [8].

An additional component done after the patient interview was completion of several health-related quality of life PROMs. These included self-rating of ECOG performance status [7], quality of life (QOL) and fatigue assessed by the Functional Assessment of Cancer Therapy - General (FACT-G) and Fatigue (FACT-F) subscale [9, 10], anxiety and depression assessed by the 12-item General Health Questionnaire (GHQ-12) [11], and the EQ-5D Thermometer Scale of overall health state [12]. In addition, patients retrospectively completed linear analogue self-assessment (LASA) scales documenting the severity of anticipatory nausea and vomiting, and nausea and vomiting within and more than $24 \mathrm{~h}$ after chemotherapy for their last cycle of chemotherapy.

Ethical approval was granted by each hospital and all participants provided written, informed consent.

\section{Statistical analysis}

The 1983 and 1993 studies had 99 and 150 patients respectively. We increased the planned sample size to 400 patients to increase the generalisability of the study and to determine if there was a difference across the number of chemotherapy cycles. Statistical analyses were conducted using Stata 13. 
Table 1 List of possible symptoms associated with chemotherapy

Group A - physical symptoms

Acne (pimples)

Ankle or legs swelling (retaining water)^

Being sick (vomiting)

Bruise easily

Burning palms and/or soles of feet ${ }^{\wedge}$

Cannot taste things

Change in the way things taste

Changes in how things smell

Changes in skin colour

Coloured urine

Constantly tired

Cough^

Deafness

Difficulty sleeping

Dry mouth^

Dry skin

Fatigue (tiredness)

Feeling sick (nausea)

Fever and/or chills^

Fingernails go brown

General aches and pains

General weakness^^

Giddiness or dizziness on standing up

Headache, migraine

Group B - non-physical symptoms

Cannot concentrate

Cannot get clothes to fit

Constant reminder of my disease ${ }^{\wedge}$

Cost of treatment^

Crying more often

Dependence on others^

Difficulty finding words ${ }^{\wedge}$

Effects my family or partner

Effects my home/work duties

Effects my social activities

Excessive time waiting for chemo^ ${ }^{\wedge}$

Fear of the future ${ }^{\wedge}$

Feeling angry

Feeling anxious, tense or worried ${ }^{\#}$

Feeling bad tempered (irritability)

Feeling like emotions are out of control^ ${ }^{\wedge}$

Feeling low, miserable (depression)

Feeling of having to have treatment which I don't think will do any good

Feeling of having to have treatment which I don't want
Heart beating faster

Hiccups $^{\wedge}$

Hot flushes

Increased appetite

Increased hair growth on legs

Increased thirst

Indigestion/reflux/discomfort ${ }^{\wedge}$

Itch $^{\wedge}$

Itching at injection site

Joint aches and pains ${ }^{\wedge}$

Joint stiffness^

Loss of appetite

Loss of hair

Loss of liquid or frequent bowel action (diarrhoea)

Loss of weight

Mouth sores (ulcers) ^

Nail changes ${ }^{\wedge}$

Nosebleeds

Not having regular bowel action (constipation)

Numbness in fingers and toes

Pain around fingernails^

Pain passing water

Pain when swallowing^

Feeling of not coping generally with treatment

Feeling overwhelmed ${ }^{\wedge}$

Feeling that the treatment is damaging my body

Feeling unattractive ${ }^{\wedge}$

Forget things

Frequency of treatment ${ }^{\wedge}$

Getting started in the mornings ${ }^{\wedge}$

Having to come to the clinic rather than a private doctor

Having to have a needle

Having to wait for treatment with other patients Infertility (cannot have children)

Lack of choice of appointment times ${ }^{\wedge}$

Lack of privacy in the chemo suite ${ }^{\wedge}$

Length of time treatment takes at clinic

Loss of independence ${ }^{\wedge}$

Loss of sexual ability

Loss of sexual feeling

Money worries^
Painful/tender veins around or above the injection site $^{\wedge}$

Passing more water than usual (increased urination)

Peeling hands and/or feet^

Periods become irregular

Periods stop

Pins and needles in fingers and toes

Ringing in ears

Runny nose ${ }^{\wedge}$

Runny or watery eyes ${ }^{\wedge}$

Shaking all over

Shortness of breath

Skin rash

Sore eyes^

Sore hands and/or feet ${ }^{\wedge}$

Sore mouth

Sore throat

Sore, tender muscles ${ }^{\wedge}$

Stuffy nose ${ }^{\wedge}$

Swollen tummy (abdominal fullness)

Thrush in your mouth^

Trouble with swallowing

Tummy ache (abdominal pain)

Weight gain

My cancer makes me different ${ }^{\wedge}$

My life is on hold^

No end to treatment ${ }^{\wedge}$

Not being able to choose where you sit for treatment ${ }^{\wedge}$

Not getting preferred place in the chemo suite ${ }^{\wedge}$

Not having the chance to ask the doctor questions

Not knowing what will happen^

Not seeing the same doctor each time

Not seeing the same nurses/staff each time ${ }^{\wedge}$

Not understanding what is happening

People looking at $\mathrm{me}^{\wedge}$

Seeing very sick people^

Slow thinking (fuzzy head)^

Thought of coming for treatment

Trouble finding somewhere to park

Trouble getting to the clinic

Unwanted advice^

Worried about my job^ ${ }^{\wedge}$

^ Additional symptoms added that were not in the original 1983 or 1993 studies

"Modified wording from original adding "or worried" 
The primary aim was to determine the most severe symptoms experienced by cancer patients while receiving chemotherapy. Descriptive statistics were used to describe the frequency of symptoms and to rank their severity. Major secondary goals included: (i) comparison of symptoms with those reported in the studies conducted in 1983 and 1993; (ii) the association of symptoms with patient characteristics such as sex, age, chemotherapy regimen (platinum-based, taxane, and other), number of prior chemotherapy cycles and PROMs.; (iii) comparison of patients' ratings of symptom severity with that of their physicians and nurses.

For the LASA scales, scores ranged from 0 (no discomfort) to 100 (extreme discomfort). Consistent with the 1993 study, a score of $>75$ was interpreted as indicating a substantial level of discomfort. Proportions were reported.

Logistic regression models were used to analyse the association of symptoms and their severity with patient demographics, chemotherapy regimen and PROMs, except for PROMs measured on a continuous scale when linear regression models were used. The regression models used the demographic, treatment or PROM variable as the dependent variable and each of the symptoms as exploratory/independent variables. Consistent with the previous studies, we restricted the analysis to comparing whether each symptom was included among the five most severe.

The 1983 study included only patients undergoing treatment for advanced cancer, whereas the 1993 study included people with earlier stage disease, so for comparison between studies we restricted analysis only to those with advanced/ metastatic cancer. Raw data were not available from the previous studies, so we compared severity rankings for symptoms.

\section{Results}

The study was completed over two time periods from 2008 to 2013 ( $n=272$ patients) and from January to October 2016 $(n=30)$ due to limitations in resources.

Of 391 patients approached, 308 consented to participate and 302 completed the assessment (Fig. 1, Consort diagram). Reasons for declining to participate were: felt too unwell; lack of interest; insufficient English; and unable to schedule.

The median age of participants was 58 years (range $17-85$ ) and $56 \%$ were female (Table 2). The predominant tumour types were colorectal (27\%), breast (22\%), and lung $(16 \%)$. Forty-five percent were being treated with curative intent. The median time since diagnosis was 6 months (range 1-392). In total, $54 \%$ of patients were receiving a platinumbased chemotherapy regimen, and $31 \%$ a taxane-containing regimen. The median number of cycles received prior to the interview was 4 (range 1-23).

\section{Symptoms experienced and severity}

Patients reported experiencing a median of 29 symptoms: 18 physical and 11 non-physical. The most common symptoms were loss of hair, general weakness, concern about effects on family or partner, loss of taste, nausea, fatigue, difficulty sleeping, concern about effects on work or home duties, and having to put life on hold. These were reported by $>50 \%$ of patients. (Table 3, Supplementary Tables 1 and 2). While other symptoms were less commonly reported, 58 of 124 symptoms were reported by $>25 \%$ of patients.

\section{Association of symptoms with patient characteristics}

Differences associated with the chemotherapy regimen, tumour type and with number of cycles were most often physical symptoms (Supplementary Tables 3 and 4). Patients on platinum-based regimens were more likely to report cough and pain when swallowing, with pins and needles, general weakness, loss of appetite and indigestion rated as more severe compared with other regimens (Supplementary Table 3 and 4). Patients receiving taxanes were more likely to report loss of hair (and to rate it as more severe), general aches and pains, and skin rash. There were no significant differences across drug regimens in the non-physical symptoms regarded as most severe.

Patients who had received more cycles of chemotherapy were more likely to report pins and needles, diarrhoea, abdominal pain, and thrush, with headaches and sore/tender muscles being rated more severe. They were also more likely to report feeling their cancer made them different. In contrast, they were less likely to report symptoms like indigestion, joint aches and pains, and ringing ears.

Women were more likely than men to report many physical and non-physical symptoms including loss of hair, pins and needles, headache, slow thinking, crying more often, and feeling unattractive. The only symptoms reported more frequently by men were passing more urine and hiccups, with trouble swallowing rated as more severe. Younger patients (under 60 years) were more likely to report concerns about the effects on work or home duties as well as difficulty sleeping and feeling angry. Older patients were more likely to report shortness of breath and easy bruising, with general weakness, changes in taste and constipation rated as more severe.

Patients treated with curative intent were more likely than those with advanced disease to report physical symptoms such as hot flushes, changes in smell, and sore eyes as well as non-physical symptoms like crying more often, seeing very sick people, and feeling of having to have treatment I don't want (Supplementary Table 3). They were also more likely to rate as severe alopecia and feeling treatment is 
Fig. 1 Consort diagram

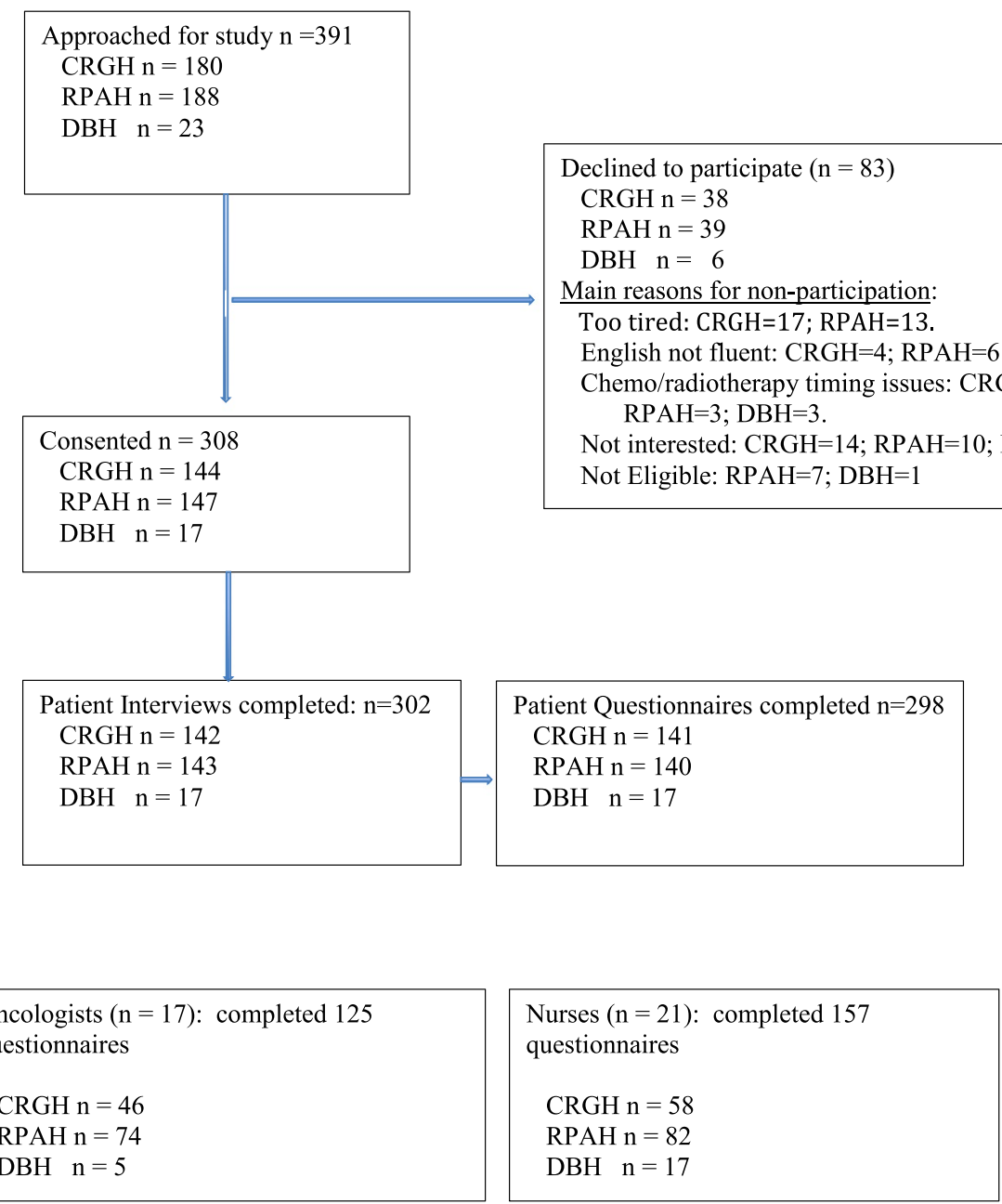

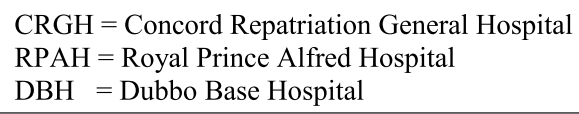

damaging (Supplementary Table 4). Patients treated with palliative intent were more likely to report loss of appetite, abdominal pain, cough, and feeling that cancer makes me different, no end to treatment and not understanding what is happening.

The most severe symptom was concern about the effects on family or partner, with $34 \%$ of patients ranking it among their five most severe symptoms and $12 \%$ as the most severe (Table 3). The next most severe symptoms were nausea, fear of the future, fatigue, not knowing what will happen, having to put my life on hold, and general weakness; these were ranked by $>15 \%$ of patients among their five most severe symptoms.

Severity of several non-physical symptoms, including having to put life on hold and cost of treatment, were associated with lower overall quality of life as measured by FACT-G and higher levels of depression and anxiety as measured by GHQ-12 (Table 4). Having to put life on hold and severe fatigue were associated with worse self-reported ECOG performance status. Other PROMS reflected well the patient reported symptoms.

\section{Comparison of patients' symptom severity with that of doctors and nurses}

Symptoms ratings by doctors and nurses were available for 96 and 116 patients respectively: their ranking of symptom severity was similar to the full sample. Perceptions of doctors and nurses matched closely the patients' own rankings (Table 3). Six of the 10 symptoms ranked most severe by patients were 
Table 2 Demographic and disease characteristics of the sample $(N=$ 302)

\begin{tabular}{|c|c|}
\hline \multicolumn{2}{|l|}{ Variable } \\
\hline Age (yrs) & $N=302$ \\
\hline Median (IQR) (Range) & $58(17)(17-85)$ \\
\hline Months from diagnosis & $N=300$ \\
\hline Median (IQR) (Range) & $6(22)(1-392)$ \\
\hline Female, n (\%) & $168(56)$ \\
\hline Marital status, n (\%) & $N=302$ \\
\hline Married/defacto & $210(70)$ \\
\hline Separated/divorced & $37(12)$ \\
\hline Single & $38(13)$ \\
\hline Widowed & $17(6)$ \\
\hline Living arrangement, $\mathrm{n}(\%)$ & $N=302$ \\
\hline Alone & $58(19)$ \\
\hline With others & $244(81)$ \\
\hline \multicolumn{2}{|l|}{ Country of birth, n (\%) } \\
\hline Australia & $198(66)$ \\
\hline Other & $104(34)$ \\
\hline Primary language, n (\%) & $N=302$ \\
\hline English & $258(85)$ \\
\hline Non-English speaking & $44(15)$ \\
\hline Education level completed, n (\%) & $N=301$ \\
\hline Primary school & $14(5)$ \\
\hline Secondary school & $129(43)$ \\
\hline College/University & $111(37)$ \\
\hline Post-graduate & 47 (16) \\
\hline Work status, n (\%) & $N=302$ \\
\hline Full-time employment & $151(50)$ \\
\hline Part-time employment & $42(14)$ \\
\hline Home duties & $9(3)$ \\
\hline Retired & $91(30)$ \\
\hline Unemployed & $9(3)$ \\
\hline Primary site of cancer, n (\%) & $N=302$ \\
\hline Colorectal & $81(27)$ \\
\hline Breast & $67(22)$ \\
\hline Lung & $49(16)$ \\
\hline Gynaecological & $30(10)$ \\
\hline Genitourinary & $16(5)$ \\
\hline Upper gastrointestinal & $13(4)$ \\
\hline Other & $46(15)$ \\
\hline Stage, n (\%) & $N=295$ \\
\hline I & $10(3)$ \\
\hline II & $25(8)$ \\
\hline III & $100(34)$ \\
\hline IV & $160(54)$ \\
\hline ECOG (patient reported), n (\%) & $N=300$ \\
\hline 0 & $52(17)$ \\
\hline 1 & $198(66)$ \\
\hline 2 & $40(13)$ \\
\hline 3 & $9(3)$ \\
\hline 4 & $1(0)$ \\
\hline
\end{tabular}

Table 2 (continued)

\begin{tabular}{ll}
\hline Other cancer treatments received, n (\%) & $N=291-299$ \\
Surgery & $214 / 299(72)$ \\
Radiotherapy & $76 / 299(25)$ \\
Endocrine & $29 / 292(10)$ \\
Other anticancer treatment(s) & $21 / 291(7)$ \\
Current chemotherapy, n (\%) & $N=296$ \\
Adjuvant & $111(38)$ \\
Neo-adjuvant & $20(7)$ \\
Metastatic & $132(45)$ \\
Palliative & $19(6)$ \\
Other & $14(5)$ \\
Current chemotherapy regimen, n (\%) & $N=296$ \\
Platinum-based & $158(54)$ \\
Taxane & $91(31)$ \\
Chemotherapy and targeted therapy & $17(6)$ \\
Current Chemotherapy cycle number & $N=291$ \\
Median (IQR) (Range) & $4(3)(1-23)$ \\
No. of lines of chemotherapy & $N=296$ \\
Median (IQR) (Range) & $1(4)(1-8)$ \\
\hline
\end{tabular}

also ranked by doctors and nurses among the 10 most severe. Having to put life on hold, difficulty sleeping, and constipation were ranked more highly by patients than doctors or nurses. Length of time spent at clinic and in waiting for chemotherapy, vomiting, and having to have a needle were ranked as more severe by doctors and nurses than by patients.

\section{Changes in symptom severity since 1983 and 1993 in people with advanced cancer}

Vomiting and nausea declined in the severity ranking across study years. Vomiting, ranked as the most severe symptom in 1983 and fifth most severe in 1993, was ranked $23^{\text {rd }}$ among symptoms of patients with advanced disease in the current study (Supplementary Table 5). Nausea, ranked as the most severe symptom in 1993, was ranked as the fifth in the current study. Conversely, concerns about effects on family increased in severity (ranked 10th in 1983, fourth in 1993 and first in the current study). Depression decreased in severity, falling to 51st in the current study while the ranking of anxiety remained more stable. Some of the non-physical symptoms that ranked highly in terms of severity in the current study, such as fear of the future, and feeling like my life is on hold, were not surveyed in the previous studies.

\section{Nausea and vomiting pre, within and post 24-h of chemotherapy}

Only $1 \%$ of patients in the current study had substantial anticipatory nausea (score of $>75$ ), and no patient reported 
Table 3 Frequency and severity of symptoms experienced by patients and comparison with severity ratings of doctors and nurses

\begin{tabular}{|c|c|c|c|c|c|}
\hline Rank & Symptom frequency (Patients) & $\begin{array}{l}\% \text { Patients } \\
\text { reporting }\end{array}$ & Symptom severity (Patients) & Symptom severity (Doctors) & Symptom severity (Nurses) \\
\hline & $N=302$ & & $N=302$ & $N=96$ & $N=115$ \\
\hline 1 & Loss of hair & 67.9 & Effects my family or partner & Fatigue & Fatigue \\
\hline 2 & General weakness & 65.9 & Nausea & Nausea & Nausea \\
\hline 3 & Effects my family or partner & 65.6 & Fear of the future & Effects my family or partner & General Weakness \\
\hline 4 & $\begin{array}{l}\text { Change in the way things } \\
\text { taste }\end{array}$ & 58.3 & Fatigue & $\begin{array}{l}\text { Not knowing what will hap- } \\
\text { pen }\end{array}$ & Effects my family or partner \\
\hline 5 & Nausea & 57.9 & $\begin{array}{l}\text { Not knowing what will hap- } \\
\text { pen }\end{array}$ & Vomiting & Alopecia \\
\hline 6 & Fatigue & 55.6 & My life is on hold & General Weakness & $\begin{array}{l}\text { Excessive time waiting for } \\
\text { chemo }\end{array}$ \\
\hline 7 & Difficulty sleeping & 52.3 & General weakness & Fear of the future & Vomiting \\
\hline 8 & Effects my work/home duties & 51.4 & Difficulty sleeping & $\begin{array}{l}\text { Length of time treatment } \\
\text { takes at clinic }\end{array}$ & Fear of the future \\
\hline 9 & My life is on hold & 50.3 & Loss of hair & Loss of appetite & Dependence on others \\
\hline 10 & Dry mouth & 49.7 & Constipation & $\begin{array}{l}\text { Constant reminder of my } \\
\text { disease }\end{array}$ & Having to have a needle \\
\hline
\end{tabular}

Table 4 Symptom severity associated with quality of life, depression and anxiety, fatigue, self-reported performance status, and overall health using linear regression models

\begin{tabular}{llll}
\hline Patient-reported outcome measure & Symptom (from interview) & Change in variable $(95 \%$ CI) & p-value \\
\hline Quality of life: & My life is on hold & $-5.8(-10.7--0.9)$ & $-9.3(-18.1--0.6)$ \\
FACT-G & Depression & $-25.1(-43.0--7.3)$ & $0.02^{*}$ \\
& Feeling unattractive & $-24.1(-42.5--5.8)$ & $0.04^{*}$ \\
& Cost of treatment & $4.7(1.6-7.9)$ & $0.01^{* *}$ \\
Depression and anxiety & Depression & $5.1(1.2-8.9)$ & $0.01^{*}$ \\
GHQ-12 & Feeling overwhelmed & $6.8(0.0-13.5)$ & $0.004^{* *}$ \\
& Cost of treatment & $-6.7(-10.0--3.3)$ \\
Fatigue & Fatigue & $-14.8(-29.6--0.1)$ & 0.01 \\
FACT-F & Cost of treatment & $4.1(1.3-13.2)$ & $0.05^{*}$ \\
Self-reported performance status (ECOG) & Fatigue & $4.1(1.4-12.2)$ & $0.05^{*}$ \\
Overall health & My life is on hold & $-6.7(-12.8--0.6)$ & $0.02^{*}$ \\
Thermometer scale & Nausea & $-9.8(-18.2--1.4)$ & $0.01^{*}$ \\
& Effects home/work duties & $-9.7(-16.1--3.4)$ \\
\hline
\end{tabular}

$* p<0.05$

$* * p<0.01$

$F A C T$, Functional Assessment of Cancer Treatment; $G$, general; $F$, fatigue subscale.

$G H Q$, General Health Questionnaire 12

ECOG, European Cooperative Oncology Group

Thermometer Scale $=$ EQ-5D Thermometer Scale of overall health state

substantial anticipatory vomiting compared with $17 \%$ and $5 \%$ in the previous studies (Table 5). Within $24 \mathrm{~h}$ of chemotherapy, $8 \%$ of patients reported substantial nausea and $4 \%$ substantial vomiting compared with $51 \%$ and $24 \%$ previously. Beyond $24 \mathrm{~h}$ after chemotherapy, $10 \%$ reported severe nausea and $4 \%$ severe vomiting compared with $57 \%$ and $29 \%$ in the previous studies. The results from LASA scales are consistent with the symptom ranking and indicate that the severity of nausea and vomiting has declined substantially since 1983.

Vomiting during the $24 \mathrm{~h}$ after chemotherapy and delayed vomiting after $24 \mathrm{~h}$ were strongly associated with higher 
Table 5 Percentage of patients with severe nausea and vomiting in relationship to timing of chemotherapy based on linear analogue selfassessment scale (LASA) rating $>75 / 100$ : for all participants, and by chemotherapy regimen

\begin{tabular}{llll}
\hline Nausea and vomiting & $\begin{array}{l}\text { Overall } \\
N=298\end{array}$ & $\begin{array}{l}\text { Platinum-based } \\
N=151\end{array}$ & $\begin{array}{l}\text { Taxane } \\
N=88\end{array}$ \\
\hline Vomiting: pre chemotherapy & 0.0 & 0.0 & 0.0 \\
Nausea: pre chemotherapy & 1.3 & 1.3 & 2.2 \\
Vomiting: within 24 h & 4.4 & 5.3 & 2.2 \\
Nausea: within 24 h & 8.4 & 7.9 & 6.7 \\
Vomiting: 24 h post chemo- & 3.7 & 5.9 & 2.2 \\
$\quad$ therapy & & & \\
Nausea: 24 h post chemo & 9.7 & 11.2 & 5.6 \\
\hline
\end{tabular}

ranking of vomiting whereas anticipatory vomiting was not. In contrast, anticipatory nausea, in addition to nausea within $24 \mathrm{~h}$ and after $24 \mathrm{~h}$ of chemotherapy, was a strong predictor of patients' nausea ranking.

\section{Discussion}

It is almost 40 years since the original 'On the Receiving End' study was published. In 1983, the top five severe symptoms were: vomiting, nausea, loss of hair, thought of coming for treatment, and length of time treatment takes, which was especially a concern for men. With improvement in management of acute physical symptoms, particularly vomiting, there has been a shift to patients reporting greater concern with non-physical side effects, particularly the impact of their cancer and/or treatment on their partner and family. In the current study, this was rated in the top five most severe symptoms by $34 \%$ and as the most severe by $12 \%$ of participants. By comparison, it was tenth in severity in 1983. Other highly rated symptoms in our study included fear of the future, uncertainty of what will happen and having to put their life on hold, which were not options in the earlier studies.

While major advances have been made in the prevention and treatment of vomiting, nausea remains difficult to manage and debilitating for patients [13]. Fatigue also continues to be a major problem with $56 \%$ of patients reporting fatigue and $66 \%$ general weakness. Our longitudinal study in colorectal cancer patients found that $70 \%$ of patients reported fatigue immediately following adjuvant chemotherapy compared with $31 \%$ who had surgery alone and $22 \%$ of healthy controls [14], with $44 \%$ still reporting fatigue 6 months after chemotherapy compared to $\sim 30 \%$ of non-chemotherapy patients and controls. Studies in women with breast cancer have reported similar results $[15,16]$.

In the present study, $45 \%$ of patients were being treated with curative intent, most with a platinum or taxane regimen, and all patients were treated in the outpatient department. In the original 1983 study, all participants had advanced cancer and many required treatment administration as inpatients. Differences were more likely to be in physical symptoms across chemotherapy regimens rather than non-physical symptoms. By comparison, the 1993 study included a third of patients being treated with adjuvant chemotherapy.

Patients are generally better prepared for physical symptoms with chemotherapy than they are for psychosocial issues. This is likely because many health care professionals focus more on physical side-effects when providing information and obtaining informed consent prior to chemotherapy [17] and in eliciting symptoms in subsequent consultations $[18,19]$. With improved physical outcomes, fear of cancer recurrence or progression, and psychosocial issues have become more salient. The similarities in non-physical symptoms ranked as severe across treatment regimens suggests the commonality of these concerns across tumour types and a need to address them more effectively. Results of the present study, and other studies showing cancer survivors have high psychological distress [20], fear of cancer recurrence, increased use of health services and poorer quality of life [21], suggest that mental health and well-being in cancer patients require greater attention.

Detecting and monitoring of symptoms experienced by patients receiving chemotherapy is an essential component of quality care. Changes in symptom profile may necessitate modification to the treatment regimen, or the provision of additional supportive care and patient education. Several studies have shown that clinicians tend to underestimate the incidence and severity of symptoms when compared to patient self-report [22-24]. Clinician accuracy in detecting patient symptoms has been reported to be lower for more subjective symptoms (e.g. fatigue and dyspnoea) than for symptoms that can be observed directly (e.g. vomiting and diarrhoea) [24]. However, in the present study, the perceptions of the oncologists and cancer nurses in rating the top five symptoms were fairly consistent with patient self-report, with oncologist awareness of troublesome non-physical symptoms greater than expected.

\section{Limitations and strengths}

Our study provides important updated information to the oncology community regarding the symptoms that patients undergoing chemotherapy experience. We acknowledge that this may be different to the symptoms they are most concerned about, and that financial concerns are likely to be much greater in countries that do not have universal health care. We did not meet our planned sample size of 400 , but recruited $\sim 300$ participants covering a broad spectrum of tumour type, chemotherapy regimens and disease stage. All patients were currently receiving chemotherapy and we 
investigated a wide array of symptoms. Data collection was spread over several years and there may have been changes in treatment and supportive care during this time which impacted symptoms. Targeted therapies and immunotherapy are not captured in this study with the exception of $6 \%$ of participants receiving a targeted therapy (e.g., trastuzumab) together with chemotherapy.

We recognise that there is confounding between some variables (for example all breast cancer patients were female, and some chemotherapy regimens are used only for certain cancer types), which limits the analysis of symptoms as a function of type of cancer, age and sex. Our matched data between patients and staff was limited to $\sim 100$ patients. Although the ranking of symptom severity by patients was similar to the larger cohort, it is possible that there was selfselection of oncologists and nurses who were more likely to discuss concerns with their patients. If so, we may have over-estimated their appreciation of symptoms most important to patients, but we would like to think that this may be due to oncology staff increasingly engaging in more patientcentred practice.

\section{Conclusions}

There has been a change in the symptoms that patients undergoing chemotherapy find most bothersome. While nausea, fatigue and general weakness remain common, the effect on family or partner was rated as the most severe, with fear of the future, not knowing what will happen, and putting my life on hold also major issues.

Supplementary Information The online version contains supplementary material available at https://doi.org/10.1007/s00520-022-06804-1.

\section{Acknowledgements In memoriam}

The team dedicate this study to Professor Martin HN Tattersall and Mrs Rhonda Devine, both deceased. Prof Tattersall was a medical oncologist who we had the honour of working with. His exceptional career placed patients at the centre of cancer care and included pioneering research into evaluating patient perspectives on cancer care, psycho-oncology, improving doctor-patient communication, and shared decision-making. Mrs Rhonda Devine was a cancer nurse consultant and clinical trials nurse we were privileged to work with. She was the living embodiment of and fierce advocate for patient-centred cancer care. Both improved the lives of people living with cancer either directly through the care they delivered or the implementation of the research they undertook.

Thanks to Shirley Lundie-Jenkins and Felicity Leslie who conducted some of the patient interviews.

Thanks to Dr Ian Tannock who reviewed the manuscript and made helpful suggestions.

We would also like to acknowledge the patients, oncology doctors and nurses from all three sites who completed the questionnaires.

Author contribution JV, HD and MHNT conceived the study and JV and HD developed the methodology. AW, IK, CO, AE, RD and CR contributed to data collection and data management. JV and HD provided supervision for the study and research team. AL performed the analysis. JV wrote the initial draft with support from AL. All living authors reviewed the manuscript, provided feedback and approved the final version.

Funding Open Access funding enabled and organized by CAUL and its Member Institutions.

Data availability Data will be shared on reasonable request to the corresponding author.

Code availability Not applicable.

\section{Declarations}

Ethics approval and consent to participate Ethical approval was granted by each hospital institutional review board prior to commencement. Written informed consent was obtained from each subject.

Consent for publication Not applicable.

Conflict of interest The authors declare no competing interests.

Open Access This article is licensed under a Creative Commons Attribution 4.0 International License, which permits use, sharing, adaptation, distribution and reproduction in any medium or format, as long as you give appropriate credit to the original author(s) and the source, provide a link to the Creative Commons licence, and indicate if changes were made. The images or other third party material in this article are included in the article's Creative Commons licence, unless indicated otherwise in a credit line to the material. If material is not included in the article's Creative Commons licence and your intended use is not permitted by statutory regulation or exceeds the permitted use, you will need to obtain permission directly from the copyright holder. To view a copy of this licence, visit http://creativecommons.org/licenses/by/4.0/.

\section{References}

1. Coates A, Abraham S, Kaye SB et al (1983) On the receiving end-patient perception of the side-effects of cancer chemotherapy. Eur J Cancer Clin Oncol 19:203-208

2. Griffin AM, Butow PN, Coates AS et al (1996) On the receiving end. V: patient perceptions of the side effects of cancer chemotherapy in 1993. Ann Oncol 7:189-195

3. Carelle N, Piotto E, Bellanger A et al (2002) Changing patient perceptions of the side effects of cancer chemotherapy. Cancer 95:155-163

4. Tran Y, Lamprell K, Nic Giolla Easpaig B et al (2019) What information do patients want across their cancer journeys? A network analysis of cancer patients' information needs. Cancer Med 8:155-164

5. Singh S, Butow P, Charles M, Tattersall MH (2010) Shared decision making in oncology: assessing oncologist behaviour in consultations in which adjuvant therapy is considered after primary surgical treatment. Health Expect 13:244-257

6. Lowy DR, Collins FS (2016) Aiming high-changing the trajectory for cancer. N Engl J Med 374:1901-1904 
7. Oken MM, Creech RH, Tormey DC et al (1982) Toxicity and response criteria of the Eastern Cooperative Oncology Group. Am J Clin Oncol 5:649-655

8. Colinet B, Jacot W, Bertrand D et al (2005) A new simplified comorbidity score as a prognostic factor in non-small-cell lung cancer patients: description and comparison with the Charlson's index. Br J Cancer 93:1098-1105

9. Cella D, Tulsky DS, Gray G et al (1993) The Functional Assessment of Cancer Therapy scale: development and validation of the general measure. J Clin Oncol 11:10

10. Yellen SB, Cella DF, Webster K et al (1997) Measuring fatigue and other anemia-related symptoms with the Functional Assessment of Cancer Therapy (FACT) measurement system. J Pain Symptom Manag 13:63-74

11. Goldberg DP. A user's guide to the General Health Questionnaire. In NFER-Nelson 1991

12. Rabin R, de Charro F (2001) EQ-5D: a measure of health status from the EuroQol Group. Ann Med 33:337-343

13. Ng TL, Hutton B, Clemons M (2015) Chemotherapy-induced nausea and vomiting: time for more emphasis on nausea? Oncologist 20:576-583

14. Vardy JL, Dhillon HM, Pond GR et al (2016) Fatigue in people with localized colorectal cancer who do and do not receive chemotherapy: a longitudinal prospective study. Ann Oncol 27:1761-1767

15. Goedendorp MM, Andrykowski MA, Donovan KA et al (2012) Prolonged impact of chemotherapy on fatigue in breast cancer survivors: a longitudinal comparison with radiotherapy-treated breast cancer survivors and noncancer controls. Cancer 118:3833-3841

16. Andrykowski MA, Donovan KA, Laronga C, Jacobsen PB (2010) Prevalence, predictors, and characteristics of off-treatment fatigue in breast cancer survivors. Cancer 116:5740-5748

17. Koedoot CG, Oort FJ, de Haan RJ et al (2004) The content and amount of information given by medical oncologists when telling patients with advanced cancer what their treatment options are. Palliative chemotherapy and watchful-waiting. Eur J Cancer 40:225-235

18. Basch E, Deal AM, Kris MG et al (2016) Symptom monitoring with patient-reported outcomes during routine cancer treatment: a randomized controlled trial. J Clin Oncol 34:557-565

19. Detmar SB, Muller MJ, Schornagel JH et al (2002) Health-related quality-of-life assessments and patient-physician communication: a randomized controlled trial. JAMA 288:3027-3034

20. Hoffman KE, McCarthy EP, Recklitis CJ, Ng AK (2009) Psychological distress in long-term survivors of adult-onset cancer: results from a national survey. Arch Intern Med 169:1274-1281

21. Butow P, Sharpe L, Thewes B et al (2018) Fear of cancer recurrence: a practical guide for clinicians. Oncology (Williston Park) 32:32-38

22. Macquart-Moulin G, Viens P, Bouscary ML et al (1997) Discordance between physicians' estimations and breast cancer patients' self-assessment of side-effects of chemotherapy: an issue for quality of care. Br J Cancer 76:1640-1645

23. Fromme EK, Eilers KM, Mori M et al (2004) How accurate is clinician reporting of chemotherapy adverse effects? A comparison with patient-reported symptoms from the Quality-of-Life Questionnaire C30. J Clin Oncol 22:3485-3490

24. Basch E, Iasonos A, McDonough T et al (2006) Patient versus clinician symptom reporting using the National Cancer Institute Common Terminology Criteria for Adverse Events: results of a questionnaire-based study. Lancet Oncol 7:903-909

Publisher's note Springer Nature remains neutral with regard to jurisdictional claims in published maps and institutional affiliations. 